\title{
COVID-19 Vaccination Coverage, Intent, Knowledge, Attitudes, and Beliefs among Essential Workers, United States
}

Kimberly H. Nguyen, David Yankey, Kelsey C. Coy, Kathryn A. Brookmeyer, Neetu Abad, Rebecca Guerin, Girija Syamlal, Peng-jun Lu, Brittney N. Baack, Hilda Razzaghi, Andrea Okun, James A. Singleton

We assessed coronavirus disease vaccination and intent and knowledge, attitudes, and beliefs among essential workers during March-June 2021. Coverage was 67\%; $18 \%$ reported no intent to get vaccinated. Primary concerns were potential side effects, safety, and lack of trust in vaccines, highlighting the importance of increasing vaccine confidence in this population.

- ssential workers, who conduct a range of operaEtions and services to ensure the continuity and viability of critical infrastructure functions, have more coronavirus disease (COVID-19) exposures and experience greater risk for severe illness and death than do nonessential workers (1-4). In December 2020, the US Advisory Committee on Immunization Practices issued recommendations prioritizing healthcare personnel (HCP), nonhealthcare frontline essential workers, and other essential workers for COVID-19 vaccination (5) (Appendix, https://wwwnc.cdc.gov/EID/ article/27/11/21-1557-App1.pdf). Previous findings indicate that $<50 \%$ of essential workers intended to get vaccinated: $37.1 \%$ in September 2020 and $49.1 \%$ in December $2020(6,7)$. Assessing vaccination coverage and intent among essential workers, who continue to face increased risk because of their public-facing roles can help tailor messages and strategies to increase vaccination uptake and confidence among this highrisk group. We analyzed data from surveys to assess COVID-19 vaccine coverage and intent and knowledge, attitudes, and beliefs (KABs) among essential workers.

Author affiliations: Centers for Disease Control and Prevention, Atlanta, Georgia, USA (K.H. Nguyen, D. Yankey, K.C. Coy, K.A. Brookmeyer, N. Abad, R. Guerin, G. Syamlal, P. Lu, B. Baack, H. Razzaghi, A. Okun, J.A. Singleton); Leidos, Inc., Atlanta (K.C. Coy)

DOI: https://doi.org/10.3201/eid2711.211557

\section{The Study}

We analyzed data from 2 nationally representative household surveys collected over 6 COVID-19 waves during March 5-June 2, 2021, Ipsos KnowledgePanel (8) and NORC AmeriSpeak (9) (Appendix). Because of the small sample sizes, to bolster the strength of the study's estimates and increase the reliability of results, we combined data for analysis from each survey during the 6 waves of data collection.

The total sample size was 7,734 respondents; 5,303 were essential workers and 2,426 nonessential workers. We used the American Association for Public Opinion Research definition for cooperation rates (10), the proportion of all respondents interviewed of all eligible units ever contacted. Among respondents, cooperation rates were $20.3 \%-60.1 \%$.

We categorized respondents as essential or nonessential workers. The essential worker category comprised the HCP, nonhealthcare frontline, and other essential worker groups (Appendix). We examined sociodemographic characteristics, including age group, sex, race and ethnicity, annual household income, health insurance status, marital status, urban versus rural status, and underlying conditions (Appendix).

We assessed vaccination status, intent, and KABs by worker group (Appendix). We categorized respondents as reachable or reluctant; reachable respondents said they probably would get or were unsure about getting a vaccine, whereas reluctant respondents said they probably or definitely would not get a vaccine. We assessed the following KABs about COVID-19 vaccination: reasons for not getting vaccinated, barriers to getting vaccinated, motivators for getting vaccinated, concerns about getting vaccinated, and concerns about vaccine side effects.

We weighted all surveys to ensure US population representation (Appendix). We used contrast 


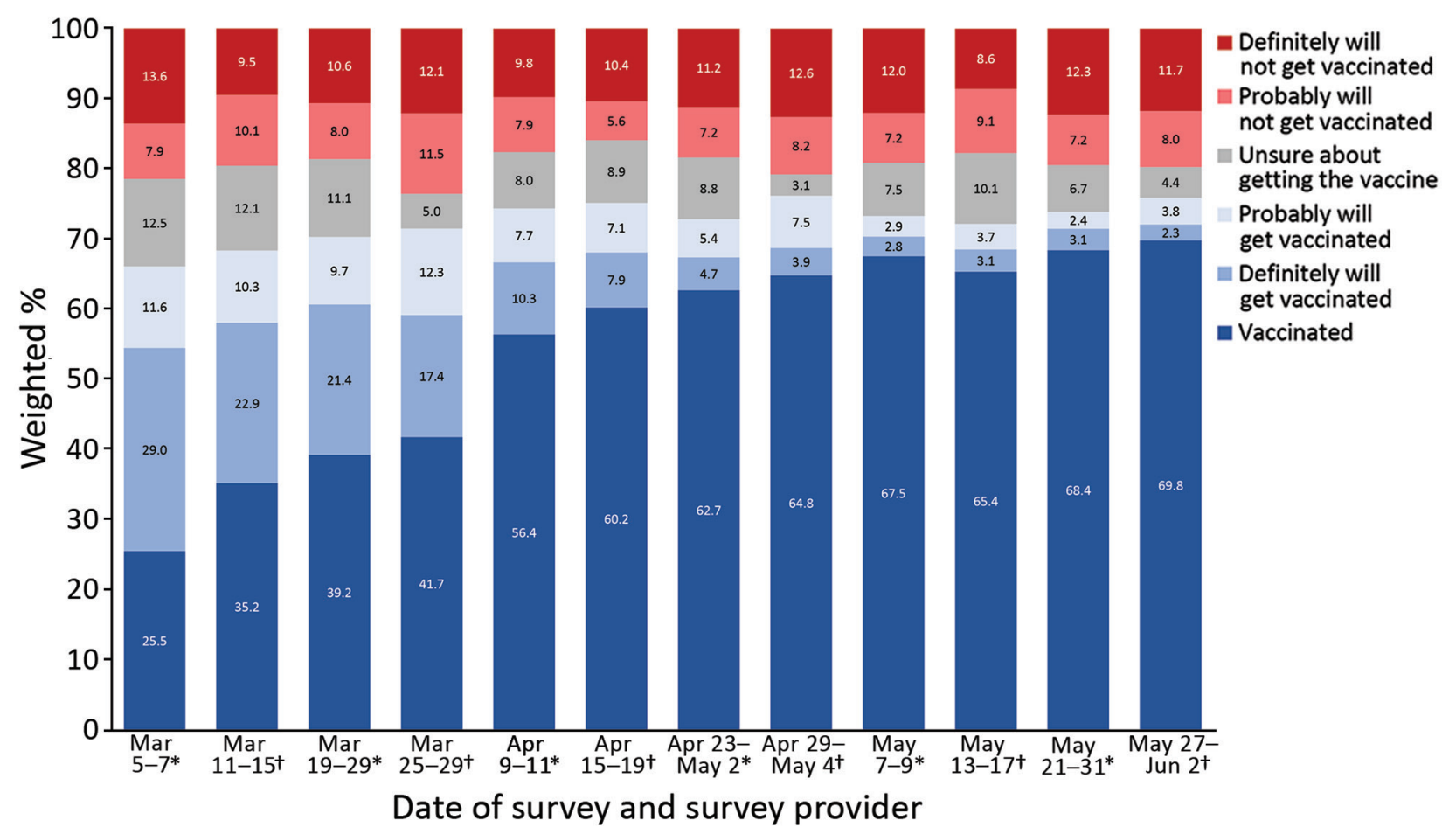

Figure 1. Trends in COVID-19 vaccination status and intent among essential workers, United States, March 5-June 2, 2021. *Data collected by Ipsos KnowledgePanel (8). †Data collected by NORC AmeriSpeak (9). COVID-19, coronavirus disease.

tests for differences in percentages to compare reachable versus reluctant groups among each of the worker categories. This activity was reviewed by the Centers for Disease Control and Prevention and was conducted consistent with applicable federal law and Centers for Disease Control and Prevention policy (Appendix).

Vaccination coverage among essential workers increased from 25.5\% in March 2021 to $69.8 \%$ in June 2021 (Figure 1). Average vaccination coverage during the

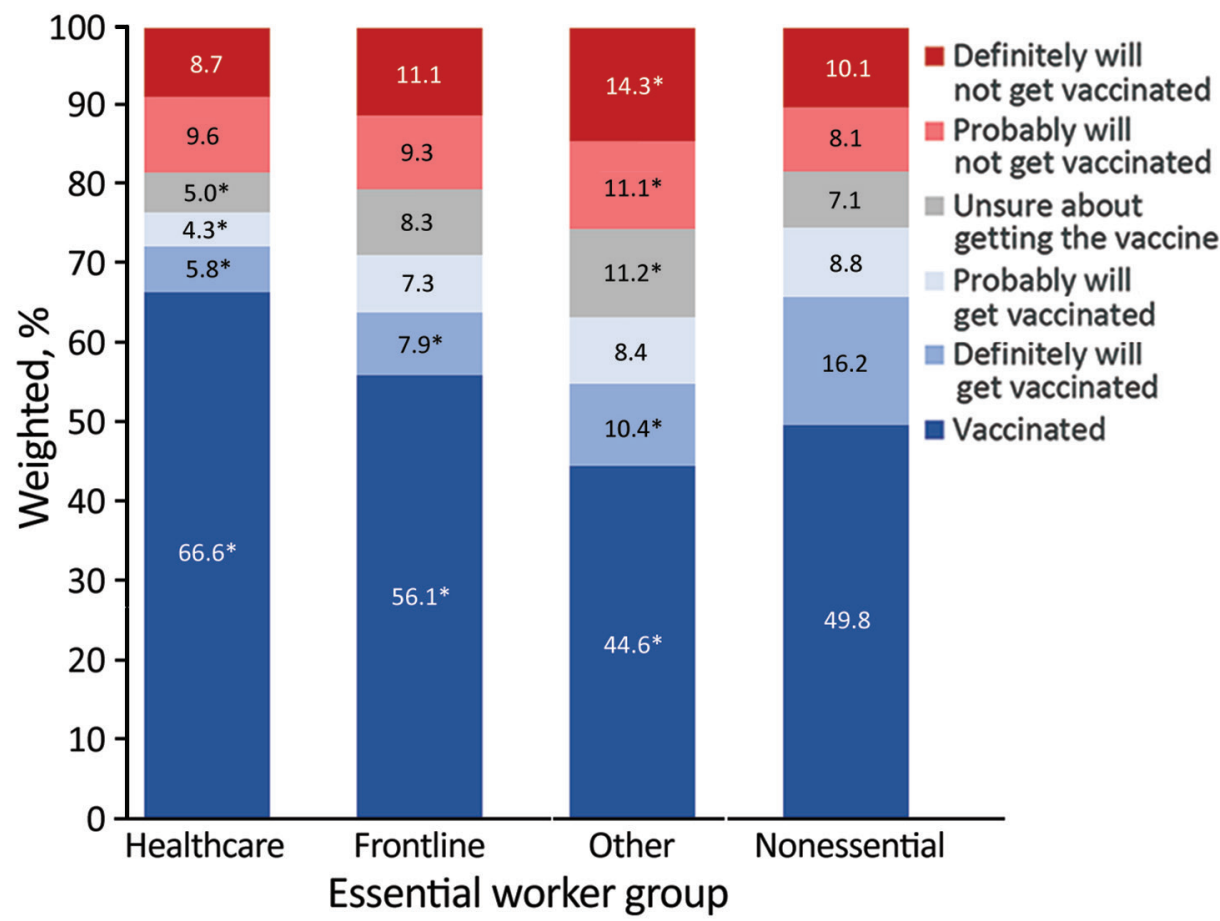

Figure 2. Average prevalence of COVID-19 vaccination status and intent by essential and nonessential worker groups, United States, March 5-June 2, 2021. Asterisk $\left({ }^{*}\right)$ indicates statistically significant $(p<0.05)$ differences between vaccination coverage and intent among each essential worker group versus vaccination coverage and vaccination intent among nonessential workers. COVID-19, coronavirus disease. 
study period was higher for $\mathrm{HCP}(66.6 \%)$ and frontline essential workers $(56.1 \%)$ and lower for other essential workers (44.6\%) and nonessential workers (49.8\%) (Figures 1,2). The percentage of reluctant persons was lowest among HCP (18.3\%) and highest among other essential workers (25.5\%) (Figure 2). In addition, the percentage of reluctant adults was highest $(25.0 \%)$ among persons 18-34 years of age; those who had a high school education or less $(28.1 \%)$, income $<\$ 25,000$ $(26.3 \%)$, or no health insurance $(32.3 \%)$; and those who lived in rural areas (29.3\%) (Appendix Table 1).

Among all unvaccinated essential workers, reasons for not getting vaccinated included concern for possible side effects $(58.0 \%)$, vaccine safety $(42.9 \%)$, and distrust of the vaccine $(41.9 \%)$ (Table 1$)$. Higher percentages of the reachable group than the reluctant group planned to wait to see if the vaccine is safe $(54.4 \%$ vs. $35.2 \%)$ and believed that other persons need the vaccine more ( $28.7 \%$ vs. $12.2 \%)$. A higher percentage of the reluctant group reported a lack of trust in COVID-19 vaccines compared with the reachable group (56.2\% vs. $22.3 \%$ ). More respondents in the reluctant group also did not believe a vaccine is needed $(36.6 \%$ vs. $10.5 \%$ of reachable group), did not think COVID-19 is much of a threat $(25.9 \%$ vs. $8.6 \%)$, and did not know whether a vaccine will work $(27.8 \%$ vs. $19.9 \%)$.

Concern about COVID-19 disease was lower $(38.5 \%)$ than concern about side effects from the vaccine $(46.4 \%)$ among all essential workers (Appendix Table 2). Among reachable groups, $42.5 \%$ reported concern about getting COVID-19 compared with $21.8 \%$ of those in reluctant groups. Among HCP,

Table 1. Reasons for not getting a COVID-19 vaccine, by essential worker group and vaccination intent, United States, MarchJune $2021^{*}$

\begin{tabular}{|c|c|c|c|c|c|c|c|c|c|c|c|c|}
\hline \multirow[b]{2}{*}{ Reason } & \multicolumn{3}{|c|}{ All groups, $n=5,308$} & \multicolumn{3}{|c|}{$\begin{array}{c}\text { Healthcare personnel, } \\
n=1,308\end{array}$} & \multicolumn{3}{|c|}{$\begin{array}{c}\text { Frontline workers, } \\
n=2,300\end{array}$} & \multicolumn{3}{|c|}{ Other workers, $n=1,700$} \\
\hline & Total & $\begin{array}{c}\mathrm{R}, \mathrm{n}= \\
714\end{array}$ & $\begin{array}{c}\mathrm{RL}, \mathrm{n}= \\
1,059\end{array}$ & Total & $\begin{array}{c}\mathrm{R}, \mathrm{n}= \\
121\end{array}$ & $\begin{array}{c}\mathrm{RL}, \mathrm{n}= \\
197\end{array}$ & Total & $\begin{array}{c}\mathrm{R}, \mathrm{n}= \\
316\end{array}$ & $\begin{array}{l}\mathrm{RL}, \mathrm{n} \\
=437\end{array}$ & Total & $\begin{array}{l}\mathrm{R}, \mathrm{n}= \\
277\end{array}$ & $\begin{array}{c}\mathrm{RL}, \mathrm{n}= \\
425\end{array}$ \\
\hline $\begin{array}{l}\text { Possible side } \\
\text { effects }\end{array}$ & $\begin{array}{c}58.0 \\
(55.0- \\
60.9) \\
\end{array}$ & $\begin{array}{c}59.5 \\
(54.7- \\
64.2) \\
\end{array}$ & $\begin{array}{c}57.3 \\
(53.4- \\
61.1)\end{array}$ & $\begin{array}{c}58.3 \\
(50.6- \\
65.7)\end{array}$ & $\begin{array}{c}55.5 \\
(44.1- \\
66.4) \\
\end{array}$ & $\begin{array}{c}60.4 \\
(50.2- \\
69.9)\end{array}$ & $\begin{array}{c}57.9 \\
(53.3- \\
62.3) \\
\end{array}$ & $\begin{array}{c}58.4 \\
(51.0- \\
65.5) \\
\end{array}$ & $\begin{array}{c}57.6 \\
(51.5- \\
63.5) \\
\end{array}$ & $\begin{array}{c}58.0 \\
(53.3- \\
62.6)\end{array}$ & $\begin{array}{r}62.3 \\
(54.5- \\
69.7)\end{array}$ & $\begin{array}{c}55.1 \\
(49.2- \\
60.9)\end{array}$ \\
\hline $\begin{array}{l}\text { Wait and see if it is } \\
\text { safe }\end{array}$ & $\begin{array}{c}42.9 \\
(40.1- \\
45.8)\end{array}$ & $\begin{array}{c}54.4 \\
(49.5- \\
59.3)\end{array}$ & $\begin{array}{c}35.2 \\
(31.6- \\
38.9)\end{array}$ & $\begin{array}{c}46.3 \\
(39.1- \\
53.7)\end{array}$ & $\begin{array}{c}57.7 \\
(46.3- \\
68.5) \\
\end{array}$ & $\begin{array}{c}41.0 \\
(31.6- \\
50.9)\end{array}$ & $\begin{array}{c}41.4 \\
(37.3- \\
45.7)\end{array}$ & $\begin{array}{c}50.4 \\
(43.5- \\
57.3) \\
\end{array}$ & $\begin{array}{c}35.0 \\
(29.9- \\
40.4) \\
\end{array}$ & $\begin{array}{c}43.0 \\
(38.2- \\
47.9)\end{array}$ & $\begin{array}{c}57.6 \\
(49.2- \\
65.7) \\
\end{array}$ & $\begin{array}{c}32.1 \\
(26.9- \\
37.8) \\
\end{array}$ \\
\hline $\begin{array}{l}\text { Do not trust the } \\
\text { vaccine }\end{array}$ & $\begin{array}{c}41.9 \\
(39.0- \\
44.8) \\
\end{array}$ & $\begin{array}{c}22.3 \\
(18.6- \\
26.4) \\
\end{array}$ & $\begin{array}{c}56.2 \\
(52.3- \\
59.9) \\
\end{array}$ & $\begin{array}{c}45.2 \\
(37.7- \\
52.8) \\
\end{array}$ & $\begin{array}{c}21.2 \\
(13.3- \\
31.1) \\
\end{array}$ & $\begin{array}{c}57.9 \\
(48.2- \\
67.2) \\
\end{array}$ & $\begin{array}{c}41.3 \\
(37.0- \\
45.6) \\
\end{array}$ & $\begin{array}{c}24.9 \\
(19.1- \\
31.5)\end{array}$ & $\begin{array}{c}54.2 \\
(48.2- \\
60.2) \\
\end{array}$ & $\begin{array}{c}41.0 \\
(36.6- \\
45.5) \\
\end{array}$ & $\begin{array}{c}19.8 \\
(14.4- \\
26.3) \\
\end{array}$ & $\begin{array}{c}57.3 \\
(51.4- \\
63.0) \\
\end{array}$ \\
\hline $\begin{array}{l}\text { Vaccine is not } \\
\text { needed }\end{array}$ & $\begin{array}{c}25.7 \\
(23.1- \\
28.5) \\
\end{array}$ & $\begin{array}{l}10.5 \\
(7.8- \\
13.7)\end{array}$ & $\begin{array}{c}36.6 \\
(32.8- \\
40.5) \\
\end{array}$ & $\begin{array}{c}23.7 \\
(17.3- \\
31.1) \\
\end{array}$ & $\begin{array}{l}8.5 \\
(4.2- \\
15.0)\end{array}$ & $\begin{array}{c}31.8 \\
(22.6- \\
42.1) \\
\end{array}$ & $\begin{array}{c}24.2 \\
(20.5- \\
28.2) \\
\end{array}$ & $\begin{array}{l}10.8 \\
(7.1- \\
15.6)\end{array}$ & $\begin{array}{c}34.4 \\
(29.0- \\
40.2)\end{array}$ & $\begin{array}{c}28.3 \\
(24.1- \\
32.8) \\
\end{array}$ & $\begin{array}{l}10.9 \\
(6.3- \\
17.2)\end{array}$ & $\begin{array}{c}41.8 \\
(36.0- \\
47.7)\end{array}$ \\
\hline $\begin{array}{l}\text { Concern about } \\
\text { allergic reaction }\end{array}$ & $\begin{array}{c}25.0 \\
(22.6- \\
27.5) \\
\end{array}$ & $\begin{array}{c}26.7 \\
(22.8- \\
31.0) \\
\end{array}$ & $\begin{array}{c}23.9 \\
(20.8- \\
27.2) \\
\end{array}$ & $\begin{array}{c}23.0 \\
(17.7- \\
29.0) \\
\end{array}$ & $\begin{array}{c}32.6 \\
(22.5- \\
44.1) \\
\end{array}$ & $\begin{array}{c}17.3 \\
(11.9- \\
24.0) \\
\end{array}$ & $\begin{array}{c}26.1 \\
(22.4- \\
30.0) \\
\end{array}$ & $\begin{array}{c}23.5 \\
(18.3- \\
29.4) \\
\end{array}$ & $\begin{array}{c}28.4 \\
(23.2- \\
33.9) \\
\end{array}$ & $\begin{array}{c}24.9 \\
(20.8- \\
29.2) \\
\end{array}$ & $\begin{array}{c}28.1 \\
(21.3- \\
35.8) \\
\end{array}$ & $\begin{array}{c}22.6 \\
(18.0- \\
27.7) \\
\end{array}$ \\
\hline $\begin{array}{l}\text { Vaccine might not } \\
\text { work }\end{array}$ & $\begin{array}{c}24.3 \\
(21.8- \\
27.0) \\
\end{array}$ & $\begin{array}{c}19.9 \\
(16.3- \\
23.9) \\
\end{array}$ & $\begin{array}{c}27.8 \\
(24.2- \\
31.5) \\
\end{array}$ & $\begin{array}{c}28.5 \\
(21.6- \\
36.2) \\
\end{array}$ & $\begin{array}{c}17.7 \\
(11.1- \\
26.3) \\
\end{array}$ & $\begin{array}{c}34.3 \\
(24.8- \\
44.8) \\
\end{array}$ & $\begin{array}{c}22.6 \\
(19.3- \\
26.3) \\
\end{array}$ & $\begin{array}{c}17.1 \\
(12.3- \\
22.9) \\
\end{array}$ & $\begin{array}{c}27.1 \\
(22.3- \\
32.4) \\
\end{array}$ & $\begin{array}{c}24.2 \\
(20.2- \\
28.6) \\
\end{array}$ & $\begin{array}{c}23.8 \\
(17.6- \\
30.9) \\
\end{array}$ & $\begin{array}{c}24.8 \\
(19.6- \\
30.5)\end{array}$ \\
\hline $\begin{array}{l}\text { Others need } \\
\text { vaccine more }\end{array}$ & $\begin{array}{c}19.0 \\
(16.8- \\
21.4)\end{array}$ & $\begin{array}{c}28.7 \\
(24.8- \\
32.9)\end{array}$ & $\begin{array}{l}12.2 \\
(9.8- \\
14.9)\end{array}$ & $\begin{array}{l}14.9 \\
(9.8- \\
21.3)\end{array}$ & $\begin{array}{c}19.7 \\
(11.9- \\
29.8)\end{array}$ & $\begin{array}{l}12.6 \\
(6.3- \\
21.7)\end{array}$ & $\begin{array}{c}18.2 \\
(15.1- \\
21.6)\end{array}$ & $\begin{array}{c}26.0 \\
(20.1- \\
32.5)\end{array}$ & $\begin{array}{l}12.4 \\
(9.2- \\
16.1)\end{array}$ & $\begin{array}{c}21.8 \\
(18.1- \\
26.0)\end{array}$ & $\begin{array}{c}35.1 \\
(27.9- \\
42.9)\end{array}$ & $\begin{array}{l}11.8 \\
(8.3- \\
16.2)\end{array}$ \\
\hline $\begin{array}{l}\text { COVID-19 is not a } \\
\text { threat }\end{array}$ & $\begin{array}{c}18.7 \\
(16.5- \\
21.0)\end{array}$ & $\begin{array}{c}8.6 \\
(5.9- \\
12.0)\end{array}$ & $\begin{array}{c}25.9 \\
(22.9- \\
29.2)\end{array}$ & $\begin{array}{c}16.3 \\
(11.0- \\
22.8)\end{array}$ & $\begin{array}{c}3.1 \\
(0.8- \\
7.9) \dagger\end{array}$ & $\begin{array}{c}23.3 \\
(15.5- \\
32.6)\end{array}$ & $\begin{array}{c}17.6 \\
(14.6- \\
21.0)\end{array}$ & $\begin{array}{l}10.4 \\
(6.2- \\
16.1)\end{array}$ & $\begin{array}{c}23.2 \\
(18.9- \\
28.0)\end{array}$ & $\begin{array}{c}21.0 \\
(17.4- \\
24.9)\end{array}$ & $\begin{array}{c}8.6 \\
(4.3- \\
15.0)\end{array}$ & $\begin{array}{c}30.4 \\
(25.3- \\
35.9)\end{array}$ \\
\hline Do not like needles & $\begin{array}{l}10.4 \\
(8.6- \\
12.5)\end{array}$ & $\begin{array}{l}12.3 \\
(9.1- \\
16.1)\end{array}$ & $\begin{array}{c}9.2 \\
(6.9- \\
11.9)\end{array}$ & $\begin{array}{c}8.7 \\
(4.5- \\
14.8)\end{array}$ & $\begin{array}{c}6.6 \\
(1.7- \\
16.7) \dagger\end{array}$ & $\begin{array}{c}9.8 \\
(4.3- \\
18.4) \dagger\end{array}$ & $\begin{array}{l}11.2 \\
(8.5- \\
14.5)\end{array}$ & $\begin{array}{c}14.7 \\
(9.9- \\
20.6)\end{array}$ & $\begin{array}{c}8.8 \\
(5.6- \\
13.0)\end{array}$ & $\begin{array}{l}10.4 \\
(7.5- \\
13.9)\end{array}$ & $\begin{array}{l}11.8 \\
(7.0- \\
18.3)\end{array}$ & $\begin{array}{c}9.3 \\
(6.1- \\
13.6)\end{array}$ \\
\hline $\begin{array}{l}\text { Obstacles prevent } \\
\text { vaccination }\end{array}$ & $\begin{array}{c}5.1 \\
(3.8- \\
6.8)\end{array}$ & $\begin{array}{c}6.7 \\
(4.4- \\
9.6)\end{array}$ & $\begin{array}{c}4.0 \\
(2.4- \\
6.3)\end{array}$ & $\begin{array}{c}6.5 \\
(2.8- \\
12.6) \dagger\end{array}$ & $\begin{array}{c}7.5 \\
(3.2- \\
14.5) \dagger\end{array}$ & $\begin{array}{c}6.0 \\
(1.4- \\
15.9) \dagger\end{array}$ & $\begin{array}{c}4.9 \\
(3.2- \\
7.2)\end{array}$ & $\begin{array}{c}6.2 \\
(3.7- \\
9.7)\end{array}$ & $\begin{array}{c}3.8 \\
(1.7- \\
7.2) \dagger\end{array}$ & $\begin{array}{c}4.7 \\
(2.7- \\
7.6)\end{array}$ & $\begin{array}{c}6.8 \\
(3.0- \\
13.1) \dagger\end{array}$ & $\begin{array}{c}3.1 \\
(1.6- \\
5.5)\end{array}$ \\
\hline $\begin{array}{l}\text { Concerned about } \\
\text { cost }\end{array}$ & $\begin{array}{c}4.6 \\
(3.2- \\
6.5)\end{array}$ & $\begin{array}{c}7.2 \\
(4.5- \\
10.8)\end{array}$ & $\begin{array}{c}2.8 \\
(1.5- \\
5.0)\end{array}$ & $\begin{array}{c}3.8 \\
(1.4- \\
8.0) \dagger\end{array}$ & $\begin{array}{c}7.0 \\
(1.6- \\
18.2) \dagger\end{array}$ & $\begin{array}{c}2.1 \\
(0.4- \\
6.2) \dagger\end{array}$ & $\begin{array}{c}3.6 \\
(2.0- \\
6.0)\end{array}$ & $\begin{array}{c}5.8 \\
(3.0- \\
10.1)\end{array}$ & $\begin{array}{c}2.0 \\
(0.5- \\
5.3)\end{array}$ & $\begin{array}{c}6.2 \\
(3.4- \\
10.2)\end{array}$ & $\begin{array}{c}8.8 \\
(3.9- \\
16.7) \dagger\end{array}$ & $\begin{array}{c}4.2 \\
(1.5- \\
9.1) \dagger\end{array}$ \\
\hline $\begin{array}{l}\text { Community } \\
\text { members are not } \\
\text { getting vaccinated }\end{array}$ & $\begin{array}{c}3.1 \\
(2.2- \\
4.2)\end{array}$ & $\begin{array}{c}2.6 \\
(1.6- \\
4.1)\end{array}$ & $\begin{array}{c}3.4 \\
(2.2- \\
5.1)\end{array}$ & $\begin{array}{c}1.2 \\
(0.3- \\
3.2)\end{array}$ & $\begin{array}{c}1.3 \\
(0.1- \\
5.5) \dagger\end{array}$ & $\begin{array}{c}1.2 \\
(0.2- \\
3.9)\end{array}$ & $\begin{array}{c}4.0 \\
(2.5- \\
6.1)\end{array}$ & $\begin{array}{c}2.9 \\
(1.3- \\
5.4)\end{array}$ & $\begin{array}{c}4.9 \\
(2.6- \\
8.4)\end{array}$ & $\begin{array}{c}2.9 \\
(1.7- \\
4.6)\end{array}$ & $\begin{array}{c}2.9 \\
(1.3- \\
5.6)\end{array}$ & $\begin{array}{c}3.0 \\
(1.3- \\
5.6)\end{array}$ \\
\hline
\end{tabular}


Table 2. Motivators for getting a COVID-19 vaccine, by essential worker group and vaccination intent, United States, MarchJune $2021^{*}$

\begin{tabular}{|c|c|c|c|c|c|c|c|c|c|c|c|c|}
\hline \multirow[b]{2}{*}{ Reason } & \multicolumn{3}{|c|}{ All groups, $n=5,308$} & \multicolumn{3}{|c|}{$\begin{array}{l}\text { Healthcare personnel, } \\
\qquad n=1,308\end{array}$} & \multicolumn{3}{|c|}{$\begin{array}{c}\text { Frontline workers, } \\
n=2,300\end{array}$} & \multicolumn{3}{|c|}{ Other workers, $n=1,700$} \\
\hline & Total $\dagger$ & $\begin{array}{c}\mathrm{R}, \mathrm{n}= \\
714\end{array}$ & $\begin{array}{c}\mathrm{RL}, \mathrm{n}= \\
1,059\end{array}$ & Total & $\begin{array}{c}\mathrm{R}, \mathrm{n}= \\
121\end{array}$ & $\begin{array}{c}\mathrm{RL}, \mathrm{n}= \\
197\end{array}$ & Total & $\begin{array}{c}\mathrm{R}, \mathrm{n}= \\
316\end{array}$ & $\begin{array}{c}\mathrm{RL}, \mathrm{n}= \\
437\end{array}$ & Total & $\begin{array}{c}\mathrm{R}, \mathrm{n}= \\
277\end{array}$ & $\begin{array}{c}\mathrm{RL}, \mathrm{n}= \\
425\end{array}$ \\
\hline $\begin{array}{l}\text { Prevent COVID- } \\
19 \text { spread to } \\
\text { family and friends }\end{array}$ & $\begin{array}{c}51.4 \\
(49.6- \\
53.2) \\
\end{array}$ & $\begin{array}{c}26.0 \\
(22.0- \\
30.3)\end{array}$ & $\begin{array}{c}4.7 \\
(3.1- \\
6.9) \\
\end{array}$ & $\begin{array}{c}58.0 \\
(54.4- \\
61.4)\end{array}$ & $\begin{array}{c}30.5 \\
(20.3- \\
42.3) \\
\end{array}$ & $\begin{array}{c}7.7 \\
(2.5- \\
17.2) \dagger\end{array}$ & $\begin{array}{c}50.5 \\
(47.7- \\
53.3)\end{array}$ & $\begin{array}{c}30.7 \\
(24.4- \\
37.5) \\
\end{array}$ & $\begin{array}{c}4.1 \\
(2.2- \\
6.8)\end{array}$ & $\begin{array}{c}47.6 \\
(44.7- \\
50.5) \\
\end{array}$ & $\begin{array}{c}19.1 \\
(13.7- \\
25.4)\end{array}$ & $\begin{array}{c}3.7 \\
(2.0- \\
6.3)\end{array}$ \\
\hline $\begin{array}{l}\text { More information } \\
\text { on vaccine } \\
\text { effectiveness }\end{array}$ & $\begin{array}{c}44.4 \\
(42.7- \\
46.2)\end{array}$ & $\begin{array}{c}31.1 \\
(26.7- \\
35.7)\end{array}$ & $\begin{array}{c}14.1 \\
(11.6- \\
16.9)\end{array}$ & $\begin{array}{c}46.0 \\
(42.6- \\
49.4)\end{array}$ & $\begin{array}{c}26.4 \\
(17.6- \\
36.8)\end{array}$ & $\begin{array}{l}13.7 \\
(8.5- \\
20.5)\end{array}$ & $\begin{array}{c}44.9 \\
(42.3- \\
47.5)\end{array}$ & $\begin{array}{c}29.1 \\
(23.5- \\
35.2)\end{array}$ & $\begin{array}{c}17.1 \\
(13.1- \\
21.7)\end{array}$ & $\begin{array}{c}42.6 \\
(39.7- \\
45.6)\end{array}$ & $\begin{array}{c}35.0 \\
(27.7- \\
42.9)\end{array}$ & $\begin{array}{l}11.0 \\
(7.4- \\
15.6)\end{array}$ \\
\hline $\begin{array}{l}\text { Reduce COVID- } \\
19 \text { spread in } \\
\text { community }\end{array}$ & $\begin{array}{c}41.9 \\
(40.2- \\
43.6)\end{array}$ & $\begin{array}{c}19.4 \\
(15.9- \\
23.2)\end{array}$ & $\begin{array}{c}2.9 \\
(1.7- \\
4.7)\end{array}$ & $\begin{array}{c}45.8 \\
(42.3- \\
49.4)\end{array}$ & $\begin{array}{l}14.5 \\
(8.3- \\
23.0)\end{array}$ & $\begin{array}{l}3.0 \\
(1.1- \\
6.5) \dagger\end{array}$ & $\begin{array}{c}42.5 \\
(39.8- \\
45.2)\end{array}$ & $\begin{array}{c}20.9 \\
(16.0- \\
26.6)\end{array}$ & $\begin{array}{c}3.2 \\
(1.0- \\
7.3) \dagger\end{array}$ & $\begin{array}{c}37.9 \\
(35.0- \\
40.9)\end{array}$ & $\begin{array}{c}19.4 \\
(13.5- \\
26.4)\end{array}$ & $\begin{array}{c}2.6 \\
(1.1- \\
5.2)\end{array}$ \\
\hline $\begin{array}{l}\text { Ability to resume } \\
\text { social activities }\end{array}$ & $\begin{array}{c}36.7 \\
(35.0- \\
38.3)\end{array}$ & $\begin{array}{c}15.5 \\
(12.3- \\
19.2)\end{array}$ & $\begin{array}{c}2.2 \\
(1.3- \\
3.3)\end{array}$ & $\begin{array}{c}38.5 \\
(35.1- \\
42.0)\end{array}$ & $\begin{array}{l}9.0 \\
(4.5- \\
15.7)\end{array}$ & $\begin{array}{c}1.2 \\
(0.2- \\
3.8)\end{array}$ & $\begin{array}{c}37.7 \\
(35.3- \\
40.2)\end{array}$ & $\begin{array}{l}14.3 \\
(9.9- \\
19.6)\end{array}$ & $\begin{array}{c}3.6 \\
(1.9- \\
6.1)\end{array}$ & $\begin{array}{c}33.8 \\
(31.1- \\
36.5)\end{array}$ & $\begin{array}{c}19.3 \\
(13.4- \\
26.3)\end{array}$ & $\begin{array}{c}1.2 \\
(0.4- \\
2.8)\end{array}$ \\
\hline $\begin{array}{l}\text { More severe } \\
\text { COVID-19 cases }\end{array}$ & $\begin{array}{c}33.4 \\
(31.8- \\
35.0)\end{array}$ & $\begin{array}{c}13.7 \\
(10.8- \\
17.0)\end{array}$ & $\begin{array}{r}4.8 \\
(3.4- \\
6.6)\end{array}$ & $\begin{array}{c}36.2 \\
(32.9- \\
39.5)\end{array}$ & $\begin{array}{l}14.4 \\
(7.8- \\
23.6)\end{array}$ & $\begin{array}{c}2.2 \\
(0.7- \\
5.4)\end{array}$ & $\begin{array}{c}33.6 \\
(31.2- \\
36.1)\end{array}$ & $\begin{array}{l}13.1 \\
(9.1- \\
18.1)\end{array}$ & $\begin{array}{l}6.2 \\
(3.5- \\
10.0)\end{array}$ & $\begin{array}{c}30.9 \\
(28.3- \\
33.6)\end{array}$ & $\begin{array}{l}14.0 \\
(9.3- \\
19.9)\end{array}$ & $\begin{array}{l}4.7 \\
(2.7- \\
7.5)\end{array}$ \\
\hline Ability & $\begin{array}{c}31.3 \\
(29.8- \\
32.8) \\
\end{array}$ & $\begin{array}{c}16.9 \\
(13.8- \\
20.5) \\
\end{array}$ & $\begin{array}{c}6.9 \\
(5.1- \\
9.1) \\
\end{array}$ & $\begin{array}{c}32.5 \\
(29.2- \\
35.9) \\
\end{array}$ & $\begin{array}{l}15.0 \\
(8.1- \\
24.5) \\
\end{array}$ & $\begin{array}{c}7.4 \\
(3.2- \\
14.2) \dagger \\
\end{array}$ & $\begin{array}{c}32.4 \\
(30.1- \\
34.7) \\
\end{array}$ & $\begin{array}{c}16.4 \\
(12.1- \\
21.6) \\
\end{array}$ & $\begin{array}{c}6.4 \\
(4.1- \\
9.4) \\
\end{array}$ & $\begin{array}{c}28.8 \\
(26.2- \\
31.4) \\
\end{array}$ & $\begin{array}{c}18.3 \\
(12.7- \\
24.9) \\
\end{array}$ & $\begin{array}{l}7.1 \\
(4.3- \\
10.8) \\
\end{array}$ \\
\hline $\begin{array}{l}\text { Someone I know } \\
\text { became seriously } \\
\text { ill or died from } \\
\text { COVID-19 }\end{array}$ & $\begin{array}{c}22.1 \\
(20.8- \\
23.5)\end{array}$ & $\begin{array}{c}9.4 \\
(6.8- \\
12.6)\end{array}$ & $\begin{array}{c}6.4 \\
(4.6- \\
8.5)\end{array}$ & $\begin{array}{c}24.1 \\
(21.4- \\
27.0)\end{array}$ & $\begin{array}{l}11.7 \\
(5.6- \\
21.0) \dagger\end{array}$ & $\begin{array}{l}2.7 \\
(0.8- \\
6.6) \dagger\end{array}$ & $\begin{array}{c}21.7 \\
(19.7- \\
23.9)\end{array}$ & $\begin{array}{l}11.5 \\
(6.9- \\
17.8)\end{array}$ & $\begin{array}{c}8.0 \\
(5.2- \\
11.6)\end{array}$ & $\begin{array}{c}21.0 \\
(18.7- \\
23.4)\end{array}$ & $\begin{array}{c}6.1 \\
(3.6- \\
9.6)\end{array}$ & $\begin{array}{l}6.6 \\
(3.9- \\
10.4)\end{array}$ \\
\hline $\begin{array}{l}\text { Recommend by a } \\
\text { healthcare } \\
\text { provider }\end{array}$ & $\begin{array}{c}17.2 \\
(16.0- \\
18.5) \\
\end{array}$ & $\begin{array}{c}14.1 \\
(10.9- \\
17.9) \\
\end{array}$ & $\begin{array}{c}3.6 \\
(2.2- \\
5.5) \\
\end{array}$ & $\begin{array}{c}19.9 \\
(17.4- \\
22.6)\end{array}$ & $\begin{array}{c}13.5 \\
(6.3- \\
24.2) \dagger \\
\end{array}$ & $\begin{array}{c}3.9 \\
(0.8- \\
11.0) \dagger \\
\end{array}$ & $\begin{array}{c}16.8 \\
(14.9- \\
18.7) \\
\end{array}$ & $\begin{array}{c}15.5 \\
(10.8- \\
21.2) \\
\end{array}$ & $\begin{array}{c}4.2 \\
(2.2- \\
7.4) \\
\end{array}$ & $\begin{array}{c}15.7 \\
(13.7- \\
17.9) \\
\end{array}$ & $\begin{array}{l}12.9 \\
(7.7- \\
19.8) \\
\end{array}$ & $\begin{array}{c}2.8 \\
(1.2- \\
5.5) \\
\end{array}$ \\
\hline $\begin{array}{l}\text { Workplace or } \\
\text { school } \\
\text { requirement }\end{array}$ & $\begin{array}{c}14.9 \\
(13.8- \\
16.1) \\
\end{array}$ & $\begin{array}{c}29.1 \\
(25.0- \\
33.5)\end{array}$ & $\begin{array}{c}14.2 \\
(11.8- \\
17.0) \\
\end{array}$ & $\begin{array}{c}19.9 \\
(17.2- \\
22.9) \\
\end{array}$ & $\begin{array}{c}39.5 \\
(28.6- \\
51.2)\end{array}$ & $\begin{array}{l}10.8 \\
(6.5- \\
16.7)\end{array}$ & $\begin{array}{c}13.7 \\
(12.0- \\
15.4)\end{array}$ & $\begin{array}{c}27.2 \\
(21.6- \\
33.5) \\
\end{array}$ & $\begin{array}{c}17.1 \\
(13.0- \\
21.9) \\
\end{array}$ & $\begin{array}{c}12.8 \\
(10.8- \\
15.0) \\
\end{array}$ & $\begin{array}{c}27.4 \\
(20.9- \\
34.7) \\
\end{array}$ & $\begin{array}{l}13.0 \\
(9.4- \\
17.4)\end{array}$ \\
\hline $\begin{array}{l}\text { Vaccine safety } \\
\text { information } \\
\text { available }\end{array}$ & $\begin{array}{c}14.8 \\
(13.4- \\
16.3) \\
\end{array}$ & $\begin{array}{c}39.8 \\
(35.4- \\
44.5) \\
\end{array}$ & $\begin{array}{c}20.8 \\
(17.9- \\
24.1) \\
\end{array}$ & $\begin{array}{l}11.7 \\
(9.3- \\
14.6) \\
\end{array}$ & $\begin{array}{c}46.6 \\
(35.6- \\
57.9) \\
\end{array}$ & $\begin{array}{c}21.2 \\
(14.7- \\
28.9) \\
\end{array}$ & $\begin{array}{c}14.2 \\
(12.2- \\
16.4)\end{array}$ & $\begin{array}{c}36.8 \\
(30.5- \\
43.3) \\
\end{array}$ & $\begin{array}{c}21.7 \\
(17.1- \\
26.8) \\
\end{array}$ & $\begin{array}{c}18.0 \\
(15.2- \\
21.1) \\
\end{array}$ & $\begin{array}{c}40.7 \\
(33.3- \\
48.6) \\
\end{array}$ & $\begin{array}{c}19.7 \\
(15.1- \\
25.1) \\
\end{array}$ \\
\hline $\begin{array}{l}\text { Enables children } \\
\text { back to school }\end{array}$ & $\begin{array}{c}14.8 \\
(13.6- \\
16.0) \\
\end{array}$ & $\begin{array}{l}8.1 \\
(5.9- \\
10.9)\end{array}$ & $\begin{array}{c}1.5 \\
(0.7- \\
2.8) \\
\end{array}$ & $\begin{array}{c}16.5 \\
(13.9- \\
19.3) \\
\end{array}$ & $\begin{array}{c}12.6 \\
(5.6- \\
23.3) \dagger\end{array}$ & $\begin{array}{c}2.0 \\
(0.6- \\
5.1)\end{array}$ & $\begin{array}{c}16.0 \\
(14.2- \\
17.8)\end{array}$ & $\begin{array}{c}8.5 \\
(5.4- \\
12.4)\end{array}$ & $\begin{array}{c}2.2 \\
(0.6- \\
5.2)\end{array}$ & $\begin{array}{c}11.8 \\
(10.0- \\
13.8) \\
\end{array}$ & $\begin{array}{l}6.1 \\
(3.3- \\
10.1)\end{array}$ & $\begin{array}{c}0.4 \\
(0.0- \\
1.6) \\
\end{array}$ \\
\hline $\begin{array}{l}\text { Enables me to get } \\
\text { back to work or } \\
\text { school }\end{array}$ & $\begin{array}{c}12.4 \\
(11.2- \\
13.7)\end{array}$ & $\begin{array}{l}4.8 \\
(3.2- \\
7.0)\end{array}$ & $\begin{array}{c}1.4 \\
(0.7- \\
2.7)\end{array}$ & $\begin{array}{c}14.3 \\
(11.7- \\
17.3)\end{array}$ & $\begin{array}{l}2.2 \\
(0.4- \\
6.7) \dagger\end{array}$ & $\begin{array}{c}1.7 \\
(0.4- \\
4.6)\end{array}$ & $\begin{array}{c}14.9 \\
(13.0- \\
16.8)\end{array}$ & $\begin{array}{c}6.4 \\
(3.7- \\
10.3)\end{array}$ & $\begin{array}{c}2.0 \\
(0.5- \\
5.1)\end{array}$ & $\begin{array}{c}7.5 \\
(6.1- \\
9.2)\end{array}$ & $\begin{array}{l}4.1 \\
(1.9- \\
7.5) \dagger\end{array}$ & $\begin{array}{c}0.7 \\
(0.1- \\
2.1)\end{array}$ \\
\hline $\begin{array}{l}\text { Recommended by } \\
\text { a family member } \\
\text { or friend }\end{array}$ & $\begin{array}{c}11.9 \\
(10.8- \\
13.0)\end{array}$ & $\begin{array}{c}5.4 \\
(3.7- \\
7.7)\end{array}$ & $\begin{array}{l}1.3 \\
(0.6- \\
2.4)\end{array}$ & $\begin{array}{l}11.2 \\
(9.0- \\
13.7)\end{array}$ & $\begin{array}{c}7.6 \\
(2.3- \\
17.8) \dagger\end{array}$ & $\begin{array}{c}2.2 \\
(0.6- \\
5.3)\end{array}$ & $\begin{array}{c}13.0 \\
(11.4- \\
14.8)\end{array}$ & $\begin{array}{c}5.9 \\
(3.4- \\
9.4)\end{array}$ & $\begin{array}{c}1.7 \\
(0.5- \\
4.4)\end{array}$ & $\begin{array}{l}10.8 \\
(9.1- \\
12.6)\end{array}$ & $\begin{array}{c}4.2 \\
(2.1- \\
7.2)\end{array}$ & $\begin{array}{c}0.3 \\
(0.0- \\
1.3)\end{array}$ \\
\hline $\begin{array}{l}\text { See community } \\
\text { members getting } \\
\text { vaccinated }\end{array}$ & $\begin{array}{l}10.3 \\
(9.1- \\
11.5)\end{array}$ & $\begin{array}{c}5.2 \\
(3.4- \\
7.5)\end{array}$ & $\begin{array}{c}2.6 \\
(1.3- \\
4.6)\end{array}$ & $\begin{array}{l}10.5 \\
(8.2- \\
13.2)\end{array}$ & $\begin{array}{l}10.2 \\
(3.7- \\
21.3) \dagger\end{array}$ & $\begin{array}{c}2.1 \\
(0.6- \\
5.2)\end{array}$ & $\begin{array}{l}11.7 \\
(9.8- \\
13.8)\end{array}$ & $\begin{array}{c}5.3 \\
(3.0- \\
8.6)\end{array}$ & $\begin{array}{l}4.5 \\
(1.7- \\
9.4) \dagger\end{array}$ & $\begin{array}{c}8.2 \\
(6.7- \\
9.9)\end{array}$ & $\begin{array}{r}3.2 \\
(1.4- \\
6.0)\end{array}$ & $\begin{array}{c}0.7 \\
(0.1- \\
2.2)\end{array}$ \\
\hline $\begin{array}{l}\text { Large increase in } \\
\text { COVID-19 cases } \\
\text { in my area }\end{array}$ & $\begin{array}{c}3.1 \\
(2.5- \\
3.9) \\
\end{array}$ & $\begin{array}{l}10.1 \\
(7.7- \\
13.0) \\
\end{array}$ & $\begin{array}{c}3.2 \\
(2.0- \\
4.9) \\
\end{array}$ & $\begin{array}{c}2.3 \\
(1.2- \\
4.1) \\
\end{array}$ & $\begin{array}{c}13.5 \\
(6.4- \\
24.1) \dagger\end{array}$ & $\begin{array}{c}2.1 \\
(0.5- \\
5.3) \\
\end{array}$ & $\begin{array}{c}3.2 \\
(2.2- \\
4.4) \\
\end{array}$ & $\begin{array}{l}7.8 \\
(5.1- \\
11.4) \\
\end{array}$ & $\begin{array}{c}5.1 \\
(2.6- \\
8.8) \\
\end{array}$ & $\begin{array}{c}3.7 \\
(2.5- \\
5.4) \\
\end{array}$ & $\begin{array}{l}11.4 \\
(7.2- \\
17.1) \\
\end{array}$ & $\begin{array}{c}1.8 \\
(0.7- \\
3.7) \\
\end{array}$ \\
\hline None of the above & $\begin{array}{c}19.9 \\
(18.3- \\
21.5) \\
\end{array}$ & $\begin{array}{c}14.1 \\
(11.1- \\
17.6)\end{array}$ & $\begin{array}{c}55.9 \\
(52.1- \\
59.6) \\
\end{array}$ & $\begin{array}{c}15.6 \\
(12.3- \\
19.4)\end{array}$ & $\begin{array}{l}10.7 \\
(5.8- \\
17.7) \\
\end{array}$ & $\begin{array}{c}54.1 \\
(44.3- \\
63.6) \\
\end{array}$ & $\begin{array}{c}17.7 \\
(15.4- \\
20.1) \\
\end{array}$ & $\begin{array}{l}11.9 \\
(8.1- \\
16.7) \\
\end{array}$ & $\begin{array}{c}52.7 \\
(46.6- \\
58.7) \\
\end{array}$ & $\begin{array}{c}26.2 \\
(23.3- \\
29.2) \\
\end{array}$ & $\begin{array}{c}17.8 \\
(12.1- \\
24.9)\end{array}$ & $\begin{array}{c}60.5 \\
(54.5- \\
66.3) \\
\end{array}$ \\
\hline tim & 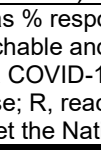 & 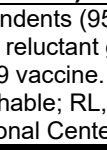 & He & 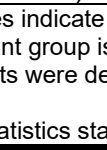 & 1 & 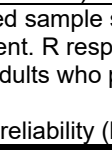 & & 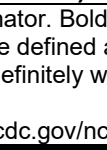 & 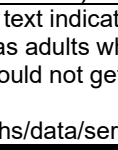 & 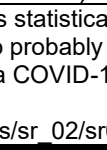 & 17 & e \\
\hline
\end{tabular}

$85.5 \%$ of reachable respondents were concerned about vaccine side effects compared with $68.8 \%$ of those in reluctant groups.

Among all essential workers, the main motivators for getting vaccinated were protection from spreading COVID-19 to family and friends (51.4\%), receiving more information on effectiveness of COVID-19 vaccines (44.4\%), and reducing spread of COVID-19 in the community $(41.9 \%)$ (Table 2 ). Motivators that were higher among the reachable than the reluctant groups were increased information on vaccine safety $(39.8 \%$ vs. $20.8 \%)$ and effica- 
cy $(31.1 \%$ vs. $14.1 \%)$, requirement by workplace or school $(29.1 \%$ vs. $14.2 \%)$, and protection for family and friends ( $26.0 \%$ vs. $4.7 \%)$.

\section{Conclusions}

Despite their increased risk for COVID-19 exposure, only about $70 \%$ of essential workers included in the sample received $\geq 1$ vaccine dose by early June 2021, similar to the $69 \%$ of all adults in the sample population during the same time period (data not shown). Over the 6 waves of data collection, HCP had the highest vaccination coverage $(66.6 \%)$; those in the other essential worker group had the lowest vaccination coverage (45\%) during March-June 2021, and one quarter were reluctant to get COVID-19 vaccinations. Consistent with another study (11), we found that younger adults and those who have lower education or income levels are more vaccine hesitant.

The first limitation of our study is that although the panel recruitment survey methodology and data weighting were designed to produce nationally representative results, respondents might not be fully representative of the general US adult population. Vaccination coverage among respondents was self-reported and could be subject to recall or social desirability bias. Data were combined across multiple survey waves, which might overaverage any recent changes in vaccination coverage and intent. Finally, state-specific vaccine prioritization varied during the data collection period, which might have affected vaccination coverage responses to items related to attitudes, behaviors, and perceptions.

Among essential workers in this sample, predominant motivators for getting vaccinated were protecting family and friends, gaining more information about the safety and effectiveness of vaccinates, and preventing community spread. These data suggest that clear, consistent messages from healthcare providers, public health officials, and immunization partners about the safety and effectiveness of the vaccine could increase vaccination coverage and vaccine confidence more broadly (12). In addition, framing messages in terms of benefits such as protecting family and friends; being able to travel; and resuming work, school, and social activities might further boost immunization coverage and confidence (12).

Among unvaccinated essential workers, nearly $60 \%$ were worried about vaccine side effects. Connecting employers and employees to credible resources on vaccine safety and expected side effects might improve vaccination coverage among essential workers. Implementing interventions to mitigate barriers to vaccination, such as flexible scheduling, paid time off for vaccination and illness resulting from side effects, on-site vaccination, and walk-in clinics, also could improve vaccination coverage.

In conclusion, our findings suggest public health officials and other leaders should differentiate between continued challenges in accessing vaccines for all populations from behavioral factors associated with vaccination. To reach vaccination goals for essential workers and everyone in the community, healthcare providers, public health officials, and immunization partners should consider KABs when tailoring messages and strategies to increase vaccination uptake and confidence, especially at local community levels.

\section{About the Author}

Dr. Nguyen is an epidemiologist in the Immunization Services Division, National Center for Immunization and Respiratory Diseases, Centers for Disease Control and Prevention (CDC), Atlanta, Georgia, USA. She is working with the CDC COVID-19 Vaccine Task Force. Her research interests include investigating gaps in COVID-19 vaccination coverage and developing strategies to increase vaccination uptake and confidence.

\section{References}

1. Cybersecurity and Infrastructure Security Agency. Guidance on the essential critical infrastructure workforce [cited 2021 May 9]. https:/ / www.cisa.gov/publication/ guidance-essential-critical-infrastructure-workforce

2. Centers for Disease Control and Prevention. Interim list of categories of essential workers mapped to standardized industry codes and titles [cited 2021 May 12]. https:/ / www. cdc.gov/vaccines/covid-19/categories-essential-workers.html

3. The Lancet. The plight of essential workers during the COVID-19 pandemic. Lancet. 2020;395:1587. https:/ / doi.org/ 10.1016/S0140-6736(20)31200-9

4. Mutambudzi M, Niedzwiedz C, Macdonald EB, Leyland A, Mair F, Anderson J, et al. Occupation and risk of severe COVID-19: prospective cohort study of 120075 UK Biobank participants. Occup Environ Med. 2021;78:307-14. https://doi.org/10.1136/oemed-2020-106731

5. Centers for Disease Control and Prevention. COVID-19 ACIP vaccine recommendations [cited 2021 Apr 21]. https://www.cdc.gov/vaccines/hcp/acip-recs/ vacc-specific/covid-19.html

6. Nguyen KH, Srivastav A, Razzaghi H, Williams W, Lindley MC, Jorgensen C, et al. COVID-19 vaccination intent, perceptions, and reasons for not vaccinating among groups prioritized for early vaccination - United States, September and December 2020. MMWR Morb Mortal Wkly Rep. 2021;70:21722. https:/ / doi.org/10.15585/mmwr.mm7006e3

7. Nguyen KH, Kahn K, Hoehner J, Hendrich M, Pedraza O, Fisun H, et al. COVID-19 vaccination intent, perceptions, and reasons for not vaccinating among groups prioritized for early vaccination, United States, September 2020 [cited 2021 Mar 29]. https://www.cdc.gov/vaccines/imz-managers/coverage/ adultvaxview/pubs-resources/COVID-online-report2020.html

8. Ipsos. KnowledgePanel: a methodological overview [cited 2021 Mar 30]. https://www.ipsos.com/sites/default/files/ ipsosknowledgepanelmethodology.pdf 
9. NORC. Amerispeak omnibus [cited 2021 Mar 30]. https:/ / amerispeak.norc.org/our-capabilities/Pages/ AmeriSpeak-Omnibus.aspx

10. American Association for Public Opinion Research. Standard definitions: final dispositions of case codes and outcome rates for surveys [cited 2021 Apr 4]. https://www.aapor. org/AAPOR_Main/media/publications/StandardDefinitions20169theditionfinal.pdf

11. Biswas N, Mustapha T, Khubchandani J, Price JH. The nature and extent of COVID-19 vaccination hesitancy in healthcare workers. J Community Health. 2021 Apr 21
[Epub ahead of print]. https:/ / doi.org/10.1007/ s10900-021-00984-3

12. Centers for Disease Control and Prevention. Building confidence in COVID-19 vaccines [cited 2021 May 15]. https://www.cdc.gov/vaccines/covid-19/vaccinate-withconfidence.html

Address for correspondence: Kimberly Nguyen, Centers for Disease Control and Prevention, 1600 Clifton Rd NE, Mailstop H24-4, Atlanta, GA 30329-4027; email: uxp1@cdc.gov

\section{January 2021}

\section{Waterborne Infections}

- Nosocomial Coronavirus Disease Outbreak Containment, Hanoi, Vietnam, March-April 2020

- Aspergillosis Complicating Severe Coronavirus Disease

- Rising Ethnic Inequalities in Acute Rheumatic Fever and Rheumatic Heart Disease, New Zealand, 2000-2018

- Differential Yellow Fever Susceptibility in New World Nonhuman Primates, Comparison with Humans, and Implications for Surveillance

- Comparative Omics Analysis of Historic and Recent Isolates of Bordetella pertussis and Effects of Genome Rearrangements on Evolution

- Performance of Nucleic Acid Amplification Tests for Detection of Severe Acute Respiratory Syndrome Coronavirus 2 in Prospectively Pooled Specimens

- Invasive Fusariosis in Nonneutropenic Patients, Spain, 2000-2015

- Hospitalization for Invasive Pneumococcal Diseases in Young Children Before Use of 13-Valent Pneumococcal Conjugate

- Estimating the Force of Infection for Dengue Virus Using Repeated Serosurveys, Ouagadougou, Burkina Faso

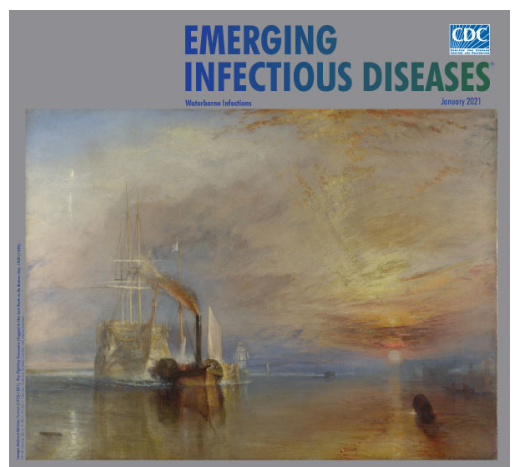

- Human Diversity of Killer Cell Immunoglobulin-Like Receptors and Human Leukocyte Antigen Class I Alleles and Ebola Virus Disease Outcomes

- IgG Seroconversion and Pathophysiology in Severe Acute Respiratory Syndrome Coronavirus 2 Infection

- Impact of Human Papillomavirus Vaccination, Rwanda and Bhutan

- Susceptibility of Domestic Swine to Experimental Infection with Severe Acute Respiratory Syndrome Coronavirus 2

- Cellular Immunity in COVID-19 Convalescents with PCR-Confirmed Infection but with Undetectable SARS-CoV-2Specific IgG

- Precise Species Identification by Whole-Genome Sequencing of Enterobacter Bloodstream Infection
- Attribution of Illnesses Transmitted by Food and Water to Comprehensive Transmission Pathways Using Structured Expert Judgment, United States

- Intrafamilial Exposure to SARS-CoV-2 Associated with Cellular Immune Response without Seroconversion, France

- Estimate of Burden and Direct Healthcare Cost of Infectious Waterborne Disease in the United States

- Post-13-Valent Pneumococcal Conjugate Vaccine Dynamics in Young Children of Serotypes Included in Candidate ExtendedSpectrum Conjugate Vaccines

- Delineating and Analyzing Locality-Level Determinants of Cholera, Haiti

- Territorywide Study of Early Coronavirus Disease Outbreak, Hong Kong, China

- Viral Metagenomic Analysis of Cerebrospinal Fluid from Patients with Acute Central Nervous System Infections of Unknown Origin, Vietnam

- Prevalence of SARS-CoV-2, Verona, Italy, April-May 2020

- Recency-Weighted Statistical Modeling Approach to Attribute Illnesses Caused by 4 Pathogens to Food Sources Using Outbreak Data, United States

\section{EMERGING} INFECTIOUS DISEASES
To revisit the January 2021 issue, go to: https://wwwnc.cdc.gov/eid/articles/issue/27/1/table-of-contents 